\section{Paroxysmal nocturnal hemoglobinuria in the differential diagnosis of thrombocytopenia}

\author{
Fusun Gediz, ${ }^{1,2}$ \\ Bahriye Kadriye Payzin, ${ }^{1}$ \\ Ozlem Zekiye Cakmak, ${ }^{3}$ Yusuf Uzum, \\ Damla Ernur, ${ }^{4}$ Fahri Sahin ${ }^{1,4}$ \\ ${ }^{1}$ Department of Hematology, Izmir \\ Katip Celebi University, Ataturk \\ Training and Research Hospital, Izmir; \\ ${ }^{2}$ PNH Education and Study Gruop; \\ ${ }^{3}$ Department of Internal Medicine Izmir \\ Katip Celebi University, Ataturk \\ Training and Research Hospital, Izmir; \\ ${ }^{4}$ Department of Hematology, Ege \\ University, Izmir, Turkey
}

\begin{abstract}
Paroxysmal nocturnal hemoglobinuria $(\mathrm{PNH})$ is a disease which diagnosis may be delayed due to variable clinical findings. We describe herein a case of $\mathrm{PNH}$ in a 21 year old woman who admitted with complaints of chronic weakness, intermittent spontaneous ecchymoses, and an intermittent abdominal pain. On laboratory tests thrombocytopenia and iron deficiency anemia without any clinical findings were found. Flow cytometric evaluations showed a PNH clone of $15 \%$ for erythrocytes, $64 \%$ for monocytes, and $60 \%$ for granulocytes. The patient was diagnosed with PNH and an eculizumab therapy was initiated. Following initiation of eculizumab therapy, the frequency of abdominal pain attacks decreased, hemoglobin level normalized, and platelet values increased slightly. In patients submitting with a triad of symptoms such as thrombocytopenia, iron deficiency anemia, and abdominal pain attacks of unknown etiology we suggest considering $\mathrm{PNH}$. We also encourage physicians to share their similar observations in order to raise the knowledge on infrequent presentations of $\mathrm{PNH}$.
\end{abstract}

\section{Introduction}

Paroxysmal nocturnal hemoglobinuria $(\mathrm{PNH})$ is a rare, life-threatening, hematological disorder characterized by hemolysis of the red blood cells due to activation of the complement system. ${ }^{1}$
The prevalence of the disease is estimated as 1 to 5 cases per million worlwide. Clinical triad of hemolytic anemia, bone marrow failure and thrombophilia is typical for $\mathrm{PNH}^{2}{ }^{2}$ The complex pathogenic mechanism of the disease is mostly discovered while some biological aspects are still under investigations. Clonal expansion of hematopoietic stem cells (HSCs) with a somatic mutation in X-linked phosphatidylinositol glycan class A $(P I G-A)$ gene are consider to be the main cause of the disease. ${ }^{3}$ Due to this mutation $\mathrm{PNH}$ is considered to be a genetic hematological disorder. However this genetic abnormality is insufficient to explain the pathogenesis of bone marrow failure and thromboembolism, therefore investigations and discussions are ongoing. ${ }^{4}$ The absence of complement regulators on erythrocytes is the main reason of chronic hemolysis patients with $\mathrm{PNH}$. Anemia, hemoglobinuria, fatigue, painful abdominal crises, dysphagia and erectile dysfunction are other symptoms related to intravascular hemolysis. ${ }^{5}$ The thromboembolism is consider to be also linked to hemolysis however the true pathophysiology is not clear yet. Thrombosis and renal failure are leading causes of death in PNH patients. ${ }^{6}$ Thrombocytopenia is a rare and unusual manifestation of $\mathrm{PNH}$. In the literature there are insufficient data about the thrombocytopenia as initial presentation of PNH. Herein we report a 21 year-old woman with $\mathrm{PNH}$ presenting with thrombocytopenia misdiagnosed as immune thromcytopenic purpura.

\section{Case Report}

Twenty-one year-old woman was reffered to our department at November 2014 from a medical center where she was followed for 2 years with diagnosis of immune thrombocytopenia and iron deficiency anemia. During that follow-up period she did not experience any bleeding and had moderate thrombocytopenia required not any treatment with antihemoragic drugs. However an oral iron supplementation on an occasional basis was given for anemia. Therefore she was referred to our clinic for further diagnosis and treatment. Her medical history showed that, she had been recovering while she was receiving oral iron supplementation; however her hemoglobin ( $\mathrm{Hb})$ levels were decreasing soon after cessation of treatment. Her thrombocytopenia (ranged from 45.000 to $55.000 / \mathrm{mm}^{3}$ ) persisted and there were no clinical findings
Correspondence: Fahri Sahin, Department of Hematology, Ege University, Bornova, Izmir, Turkey.

Tel.:+90.5323321269 - Fax:+90.2323904293. E-mail: drfahrisahin@gmail.com

Key words: thrombocytopenia, abdomina pain, paroxysmal nocturnal hemoglobinuria.

Acknowledgements: the authors would like to thank to Doğuş Medical Consulting Company for medical writing and editing assistance provided in the preparation of this article

Contributions: all authors participated in diagnosis, observation, treatment and follow-up phases of this case; FG also participated in designing and drafting the manuscript.

Conflict of interest: the authors declare no potential conflict of interests.

Received for publication: 4 September 2016. Revision received: 13 November 2016.

Accepted for publication: 17 November 2016

This work is licensed under a Creative Commons Attribution-NonCommercial 4.0 International License (CC BY-NC 4.0).

(C) Copyright F. Gediz et al., 2017

Licensee PAGEPress, Italy

Hematology Reports 2017; 9:6862

doi:10.4081/hr.2017.6862

consistent with blood loss that can explain a possible iron deficiency anemia. Also according to her anamnesis she reported complaints of chronic weakness, intermittent spontaneous ecchymoses, and an intermittent abdominal pain of unknown etiology. All necessary evaluations including abdominal ultrasound, and also assessments regarding thrombosis and FMF were performed however no clinical evidence was found for her abdominal pain.

Her thrombocytopenia (ranged from 45.000 to $55.000 / \mathrm{mm}^{3}$ ) persisted and there were no clinical findings consistent blood loss that can explain a possible iron deficiency anemia. On physical examination, she was anemic (pale appearance of skin and mucous membranes), her spleen was slightly palpable $\left(1 \mathrm{~cm}\right.$ from $12^{\text {th }}$ rib), and there were a few small (diameter $<1 \mathrm{~cm}$ ) ecchymosis at her lower extremity. During admission a series of laboratory tests and a bone marrow examination have been performed. Initial laboratory test showed a thrombocytopenia with a mild anemia and increased serum LDH levels (Table 1). Urine analysis showed hemosiderin cylinders. Bone marrow examination (both aspiration and biopsy) 
revealed just a mild erythroid hyperplasia (normal cellularity: 60\%). Hypersegmentation in megakaryocytes and and slight dysplastic changes (multiple separated nucleus) were also observed. Cytogenetics was performed and result was 46,XX. Presence of iron deficiency was observed via bone marrow biopsy performed using iron staining. Therefore we performed fluoresceinlabeled proaerolysin (FLAER) flow cytometric assay with the pro-diagnosis of non-immune mediated hemolytic anemia. Flow cytometric evaluations (with FLAER and/or CD16, CD24, CD66b) showed a PNH clone of $15 \%$ for erythrocytes, $64 \%$ for monocytes, and $60 \%$ for granulocytes. According to clinical, laboratory, and cytogenetic result the patient was diagnosed with $\mathrm{PNH}$ and eculizumab therapy was started. After initiation of eculizumab therapy, abdominal pain attacks disappeared, both hemoglobulin and LDH levels normalized, and platelet values increased slightly as expected. Also no adverse reaction was seen due to eculizumab therapy.

\section{Discussion}

Paroxysmal nocturnal hemoglobinuria is a rare, life-threatening, genetic hematological disorder characterized by hemolysis of the red blood cells. The classic manifestation of $\mathrm{PNH}$ is dark urine during the night with partial clearing during the day. ${ }^{7}$ Its mostly present with hemolytic anemia usually in the form of intravascular hemolysis. The most common presentation is the presence of anemia associated with dark cola- colored urine that is a manifestation of hemoglobinuria. ${ }^{8}$ The latter may be confused with hematuria, and erroneous treatment could be given for urosepsis. Elevated reticulocyte count and serum LDH level with a low serum haptoglobin level in the absence of hepatosplenomegaly are the hallmarks of intravascular hemolysis. The bone marrow is usually markedly erythroid, with decreased or absent iron stores, depending on how long the patient has been losing iron in the urine. ${ }^{9}$ Our case was interesting because of unusual presentation with thrombocytopenia. In the literatüre the frequency of $\mathrm{PNH}$ patients presenting with thrombocytopenia is relatively low. In this cases, it is consider that thrombocytopenia may be occur in a hypoplastic bone marrow similar to aplastic anemia (aplastic episodes). Our patient also presenting with recurrent abdominal pain. During diagnosis, $10 \%$ to $33 \%$ of patients with PNH may present with abdominal pain and other gastrointestinal disorders (dysphagia). These symptoms are more common for classical PNH patients. ${ }^{10}$ Routine screening is not mandatory for all patients presenting with these symptoms. However if clinical and laboratory features of intravascular hemolysis and/or cytopenia are observed they should be screened for PNH. ${ }^{11}$ In general clinical practice eculizumab treatment is recommended for $\mathrm{PNH}$ patients with thrombosis, portal hypertension or renal failure. ${ }^{12}$ However some studies wich aim to identify thromboembolism (TE) associated risk factors showed that, patients with elevated hemolysis (LDH levels $\geq 1.5$ times the upper limit of normal [ULN]) at diagnosis were at significantly higher risk for TE than patients with $\mathrm{LDH}$
$<1.5 \times$ ULN ${ }^{13}$ Brodsky et al. reported that patients with LDH levels $>1.5 \times$ ULN are under great risk of thrombosis. ${ }^{14}$ Furthermore the combination of $\mathrm{LDH}$ $\geq 1.5 \times$ ULN with the clinical symptoms such as abdominal pain was associated with a greater increased risk for TE than elevated hemolysis or clinical symptoms alone. According to our patient's medical history she presented high LDH levels with fluctuating manner for the last 2 years. Therefore, we decided to start eculizumab therapy because of increased PNH clone size, increased serum LDH level and increased risk of thrombosis. This may prevented thrombosis and increase the quality of life of the patient. $\mathrm{PNH}$ is a paroxysmal disease, and it clinical and laboratory findings are not so prominent between attacks. Because of this it may be difficult to make the true diagnosis, and late or misdiagnosis is highly possible. PNH is a disease which diagnosis may be delayed due to variable clinical findings and this may lead to increase the risk of mortality and morbidity. ${ }^{15}$ Early diagnosis is important for both to prevent the morbidity and to reduce the risk of mortality.

\section{Conclusions}

In conclusion, we suggest considering $\mathrm{PNH}$ diagnosis in patients submitting with a triad of symptoms: thrombocytopenia, iron deficiency anemia, and abdominal pain attacks of unknown etiology. We also encourage physicians to share their similar observations in order to raise the knowledge on this unusual presentation of PNH.

Table 1. Laboratory results of the patient before and after Eculizumab treatment.

\begin{tabular}{lcc}
\hline Parameter & Pre-treatment & 2 months post-treatment \\
White blood cells $\left(/ \mathrm{mm}^{3}\right)$ & 4800 & 6700 \\
Hemoglobin $(\mathrm{g} / \mathrm{dL})$ & 10.1 & 12.0 \\
\hline Platelets $\left(/ \mathrm{mm}^{3}\right)$ & 58,000 & 78,000 \\
Reticulocyte $(\%)$ & 5.9 & 3.2 \\
\hline Lactate dehydrogenase (U/L) & 547 & 227 \\
Direct Coombs test & $(-)$ & $(-)$ \\
\hline Mean corpuscular volume (fl) & 88 & 92 \\
Haptoglobulin (mg/dL) & 12 & 42 \\
\hline Serum ferritin levels (ng/mL) & 12 & 124 \\
Antinuclear antibodies & $(-)$ & $(-)$ \\
\hline Anti-dsDNA antibodies & $(-)$ & $(-)$ \\
HBsAg, Hepatitis C virus and HIV serology & $(-)$ & $(-)$ \\
\hline Lupus anticoagulant (lupus antibody) & $(-)$ & $(-)$
\end{tabular}

\section{References}

1. Risitano AM, Rotoli B. Paroxysmal nocturnal hemoglobinuria: pathophysiology, natural history and treatment options in the era of biological agents. Biologics 2008;2:205-22.

2. Risitano AM, Perna F, Selleri C. Achievements and limitations of complement inhibition by eculizumab in paroxysmal nocturnal hemoglobinuria: the role of complement component 3. Mini Rev Med Chem 2011;11:528-35

3. Hillmen P, Lewis SM, Bessler M, et al. Natural history of paroxysmal nocturnal hemoglobinuria. N Engl J Med 1995;333:1253-8.

4. Socié G, Mary JY, de Gramont A, et 
al. Paroxysmal nocturnal haemoglobinuria: long-term follow-up and prognostic factors. Lancet 1996;348: 573-7.

5. Lee JW Jang JH, Kim JS, et al. Clinical signs and symptoms associated with increased risk for thrombosis in patients with paroxysmal nocturnal hemoglobinuria from a Korean Registry. Int J Hematol 2013;97:74957.

6. Sahin F, Ozkan MC, Mete NG, et al. Multidisciplinary clinical management of paroxysmal nocturnal hemoglobinuria. Am J Blood Res 2015;5:1-9.

7. DeZern AE, Brodsky RA. Paroxysmal nocturnal hemoglobinuria: a complement-mediated hemoly- tic anemia. Hematol Oncol Clin North Am 2015;29:479-94.

8. Parker C, Omine M, Richards S, et al. Diagnosis and management of paroxysmal nocturnal hemoglobinuria. Blood 2005;106:3699-709.

9. Brodsky RA. Complement in hemolytic anemia. Blood 2015;126: 2459-65.

10. Sahin F, Yilmaz AF, Ozkan MC et al. PNH is a debilitating, fatal but treatable disease: same disease, different clinical presentations. Am J Blood Res 2015;5:30-3.

11. Borowitz MJ, Craig FE, Digiuseppe JA, et al. Guidelines for the diagnosis and monitoring of paroxysmal nocturnal hemoglobinuria and related disorders by flow cytometry.
Cytometry B Clin Cytom 2010;78: 211-30.

12. Parker C. Eculizumab for paroxysmal nocturnal haemoglobinuria. Lancet 2009;373:759-67.

13. Socie G, Mary JY, de Gramont A, et al. Paroxysmal nocturnal haemoglobinuria: long-term follow-up and prognostic factors. French Society of Haematology. Lancet 1996;348:5737.

14. Brodsky RA. Advances in the diagnosis and therapy of paroxysmal nocturnal hemoglobinuria. Blood Rev 2008;22:65-74.

15. Sahin F, Akay OM, Ayer M, et al. Pesg PNH diagnosis, follow-up and treatment guidelines. Am J Blood Res 2016;6:19-27. 